\title{
A Monolithic Multisensor Microchip with Complete On-Chip RF Front-End
}

\author{
Massimo Merenda ${ }^{1,2, *}$ (D), Corrado Felini ${ }^{1}$ and Francesco G. Della Corte ${ }^{1,2}$ \\ 1 Department of Information Engineering, Infrastructure and Sustainable Energy (DIIES), \\ “Mediterranea" University, Via Graziella Loc. Feo di Vito, 89124 Reggio Calabria, Italy; \\ corrado.felini@unirc.it (C.F.); francesco.dellacorte@unirc.it (F.G.D.C.) \\ 2 HWA srl-Spin Off dell'Università Mediterranea di Reggio Calabria, Via Reggio Campi II tr. 135, \\ 89126 Reggio Calabria, Italy \\ * Correspondence: massimo.merenda@unirc.it; Tel.: +39-0965-1693-441
}

Received: 9 November 2017; Accepted: 29 December 2017; Published: 2 January 2018

\begin{abstract}
In this paper, a new wireless sensor, designed for a $0.35 \mu \mathrm{m} \mathrm{CMOS} \mathrm{technology,} \mathrm{is} \mathrm{presented.}$ The microchip was designed to be placed on an object for the continuous remote monitoring of its temperature and illumination state. The temperature sensor is based on the temperature dependence of the I-V characteristics of bipolar transistors available in CMOS technology, while the illumination sensor is an integrated p-n junction photodiode. An on-chip $2.5 \mathrm{GHz}$ transmitter, coupled to a mm-sized dipole radiating element fabricated on the same microchip and made in the top metal layer of the same die, sends the collected data wirelessly to a radio receiver using an On-Off Keying (OOK) modulation pattern.
\end{abstract}

Keywords: CMOS sensors; On-Chip-Antenna; LC oscillator; temperature sensor; light sensor; Internet of Things

\section{Introduction}

Microchip sensors have the advantage that the transducers can be monolithically integrated with signal amplification and conditioning circuitry, with clear advantages in terms of reduced size, noise immunity and production costs. With the advent of the Internet-of-Things [1], these devices are expected to gain in popularity, as they can be considered the link between the world surrounding an object they are attached to and electronics. Of course, sensors that are readily deployable and can be easily accessed, possibly in a wireless manner, are highly desirable [2,3]. Nevertheless, the application of an external antenna to a microchip to allow wireless communication is a costly process and moreover leads to the de-miniaturization of the device [4]. Therefore, the concept of an on-chip-antenna (OCA), fabricated during the CMOS process, which eliminates the need for external transmission lines and sophisticated packaging, seems to be the simplest approach to warrant short range data transmission together with low costs and reliability. Usually, this task is obtained by integrating spiral inductors as the radiating elements [5-8]. In this paper, we present a full custom microchip integrating a dipole RF radiator fed by an LC oscillator and an RF power amplifier. The choice of a dipole antenna allowed the design of almost one-dimensional form factor microchips, while loop antennas require square devices. The device includes a temperature sensor, a light sensor and a general-purpose input and was designed for ambient and processes monitoring when temperature, illumination or other output signals need to be kept under control. Two examples of possible application scopes are photovoltaic cells in solar modules and light emitting diodes. By means of the integrated $2.5 \mathrm{GHz}$ transmitter, the device wirelessly sends the collected data to a base station, placed up to a few meters away. The microchip was fabricated by a silicon foundry (Austria Micro Systems), with a standard and low 
cost $0.35 \mu \mathrm{m}$ CMOS process (C35B4M3) [9], which demonstrates that even low cost technologies are suitable for this kind of fully integrated sensors.

The paper is organized as follows. In Section 2 we present the microchip architecture with a general description of its internal organization. In Section 3 we provide details about the integrated sensors, the analogue front-end, the control logic and the RF section. In Section 4 we provide experimental results on the microchip operation. Finally, the conclusions are drawn in Section 5.

\section{System Architecture}

The architecture of the proposed device is shown in Figure 1. The analogue section includes two sensors, namely a temperature sensor and a light sensor. The former is designed to provide a "proportional to absolute temperature" (PTAT) output and guarantees an optimal linearity; the latter is in fact an integrated solar cell, designed to provide a useful output under normal outdoor daylight irradiation. Sensor outputs are first amplified to bring their respective signals to useful levels and then digitalized by means of a single analogue-to-digital converter (ADC). The ADC is time-shared between either two or three sources, namely the PTAT, the light sensor and in case a generic input applied between two input pads of the microchip, where an additional external sensor can be applied. The serial conversion timing is provided by the digital section.

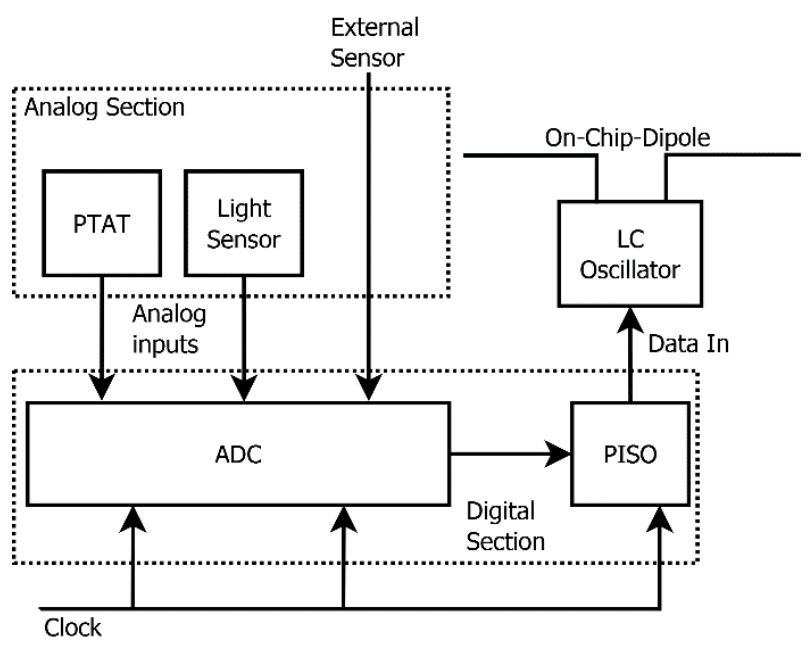

Figure 1. Wireless multisensor architecture.

The ADC serial outputs are afterwards loaded into a parallel-in/serial-output (PISO) $2 \times 8$ (or $3 \times 8)$ bits shift register. Once the measurements are done and the PISO loaded, its content is serialized for transmission at a bit-rate of $3 \mathrm{kbps}$. The microchip is also internally provided with an integrated RF transmitter, composed of an LC-oscillator, turned on and off by the bitstream and an RF power amplifier (PA), in turn energizing an integrated small dipole antenna.

\section{Microchip Blocks Description}

\subsection{Temperature Sensor}

Low cost high-performance temperature sensors are increasingly required. Besides, it is needed to reduce their power consumption. This concept is necessary to use the sensor in battery operated systems, to decrease the errors produced by self-heating and to be able to add the sensor to a chip without causing a significant increase in the power consumption of the complete system [10].

An integrated CMOS temperature sensor perfectly fits low cost, high performance and low power consumption. 
Several temperature sensors topologies have been proposed in CMOS technology. Most of the systems reported in literature, including the one presented hereafter, exploit a PTAT scheme, realized by means of coupled bipolar junction transistors (BJT) [11,12].

In fact, assuming for PNP BJTs the collector current given by:

$$
I_{C}=I_{S} e^{\left(\frac{q V_{E B}}{\eta k T}\right)},
$$

with $I_{S}$ the saturation current of the emitter-base junction, $V_{E B}$ the emitter-base voltage, $k$ the Boltzmann constant, $q$ the electronic charge and $\eta$ the ideality factor, if two identical transistors $\left(I_{S 1}=I_{S 2}=I_{S}\right)$ are biased at collector currents of $I_{r e f}$ and Io respectively, the difference between the emitter-base voltages of the two BJTs has a linear dependence on $T$, according to the following equation.

$$
\Delta V_{E B}=V_{E B 1}-V_{E B 2}=V_{t} \ln \frac{I_{r e f}}{I_{s}}-V_{t} \ln \frac{I o}{I_{s}}=\frac{k T}{q} \ln \left(\frac{I_{r e f}}{I o}\right),
$$

where $\eta=1$ is assumed.

Our sensor was designed to provide a high sensitivity and a signal level tuned in order to simplify the Analog-to-Digital conversion (ADC) in the temperature range from room temperature to $100{ }^{\circ} \mathrm{C}$.

The temperature sensor consists of two building blocks: a PTAT voltage generator and a differential amplifier, as shown in Figure 2.

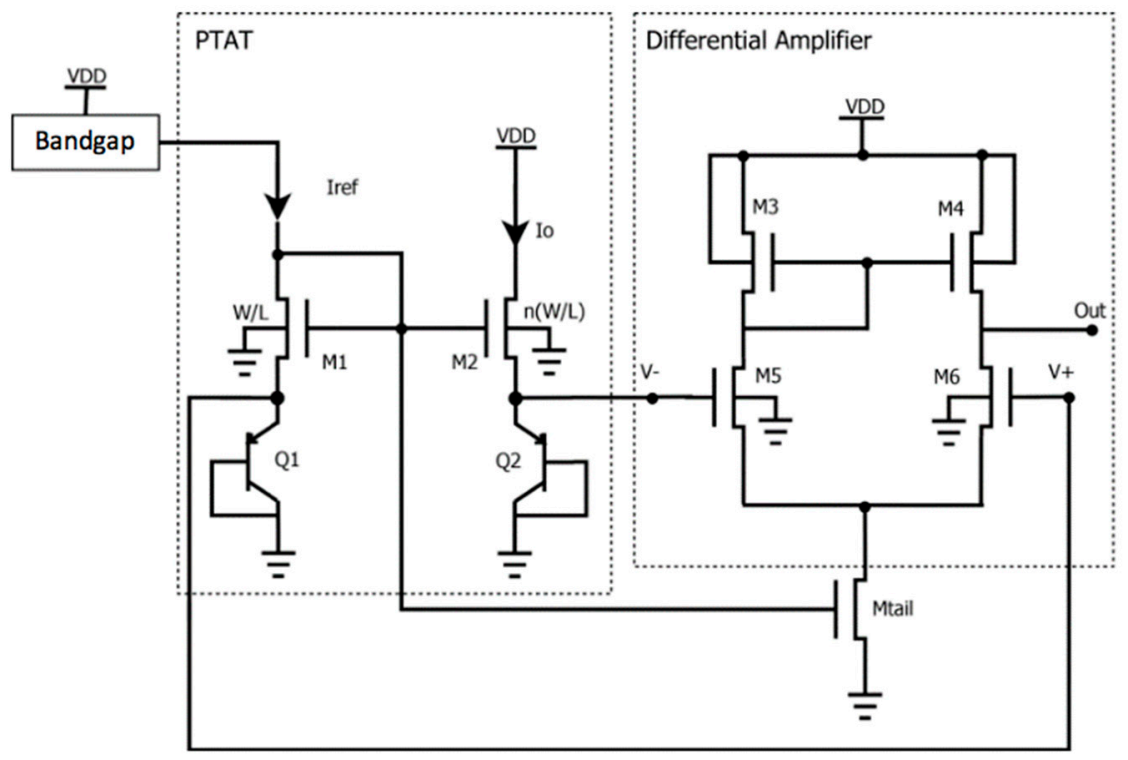

Figure 2. Architecture of the integrated PTAT with differential amplifier.

To obtain a proportional output signal with respect to absolute temperature, two diode-connected BJTs, driven by different currents, were realized. The two PNP transistors Q1 and Q2, both working in the active region, are realized side-by-side on the chip and have the same geometry and orientation and therefore can be assumed identical. The ratio between their collector currents is fixed by the different $\mathrm{W} / \mathrm{L}$ aspect ratios of $\mathrm{M} 1$ and $\mathrm{M} 2$. Assuming in principle negligible channel length modulation effects [13], Q2 is driven by a current Io that is $n \times I_{r e f}$, where $n$ is the channel width ratio $\mathrm{W}_{\mathrm{M} 1} / \mathrm{W}_{\mathrm{M} 2}$ while $\mathrm{L}_{\mathrm{M} 1}=\mathrm{L}_{\mathrm{M} 2}$. In this work $n=10$ was chosen for an appropriate sizing of the MOS transistor as trade-off between sensitivity in the measured temperature range and linearity of the output signal.

The reference current $I_{r e f}$ is generated through an integrated Bandgap current reference [14] with supply and temperature compensation that delivers a constant current of $11.5 \mu \mathrm{A}$, showing a temperature dependency of about $800 \mathrm{ppm} /{ }^{\circ} \mathrm{C}$. As shown by the simulations, this temperature 
dependency has no practical impact on the performance of the two sensors. The source is designed to supply each diode-connected BJT with currents that are independent from $V_{d d}$.

In Figure 3 the simulated emitter-base voltages of transistors Q1 and Q2 at various temperatures are shown. Their behaviour shows a negative derivative with respect to temperature. From simulations, it is possible to calculate the sensor output at each absolute temperature:

$$
V_{P T A T}(T)=V_{+}-V_{-}
$$

In the same Figure, the differential output of the PTAT in the temperature range from 0 to $100{ }^{\circ} \mathrm{C}$ is shown.

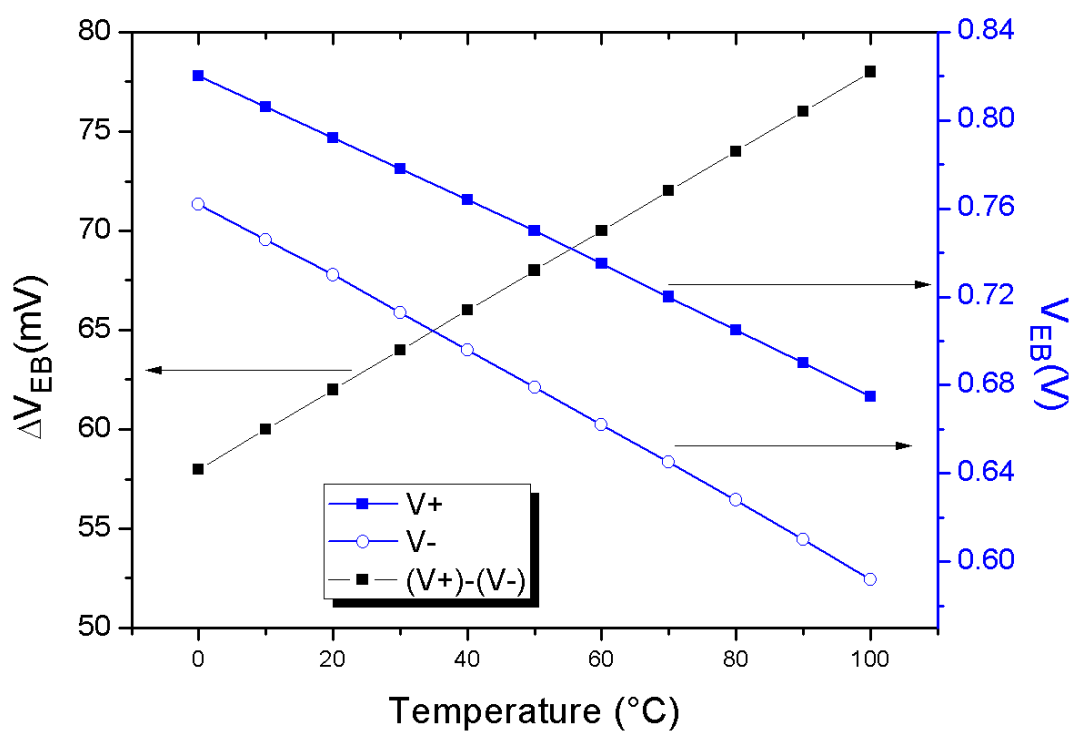

Figure 3. Emitter-base voltage of transistors Q1 and Q2 at various temperatures (right scale) and differential output of the PTAT (left scale) in the temperature range from 0 to $100{ }^{\circ} \mathrm{C}$. The lines are guides to the eye.

In the temperature range of interest, the estimated power consumption of the two PNP transistors is $7.7 \mu \mathrm{W}$ and $66.5 \mu \mathrm{W}$ respectively for Q1 and Q2, obtained from simulations. This power might cause self-heating of the sensor and therefore the PTAT circuit is activated only for a few milliseconds through the Bandgap circuit at regular intervals.

The amplifier stage was designed through extensive parametric simulations aimed at obtaining output signal levels that could be reasonably applied to the ADC, while preserving a good linearity. Here, M3 and M4 are used as active load, instead of using high value resistors, whose implementation is wasteful in terms of area consumption. M5 and M6 have been sized to achieve the maximum output dynamics for the differential amplifier. Being the PTAT output differential, the topology requested for the amplifier is differential as well. $\mathrm{M}_{\text {tail }}$ has the role of a current limiter for the amplifier, in order to reduce power consumption.

\subsection{Light Sensor}

The proposed solution for the design of the CMOS light sensor is based on planar p-n junction formed by the P-type substrate and the N-type well. This structure could be figured out as a micro solar cell producing a current proportional to surface irradiation. The outermost metal layer (Metal- 1 for the CMOS technology) was used to build fingers that collect photocurrent at the cathode. The structure of the light sensor is shown in Figure 4. 
(a)

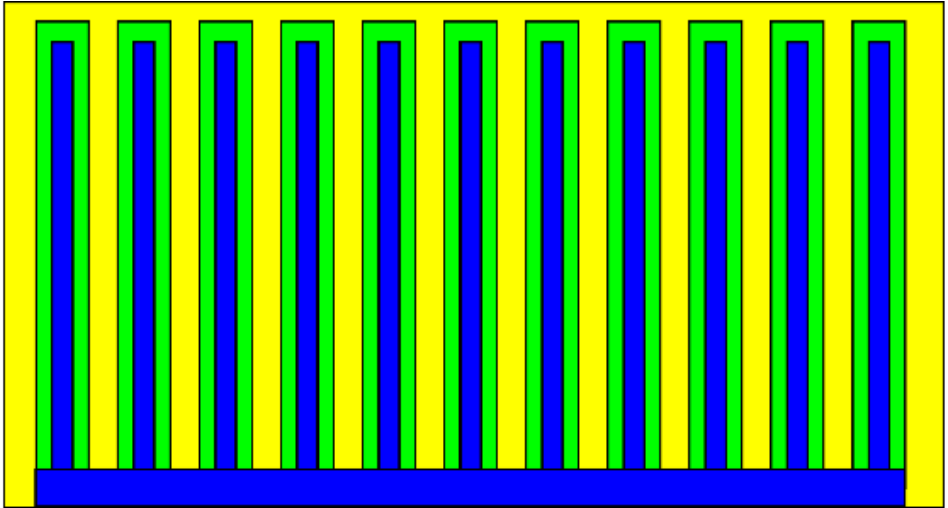

P-Substrate

$\mathrm{N}-$ Well

Metal Layer

(b)

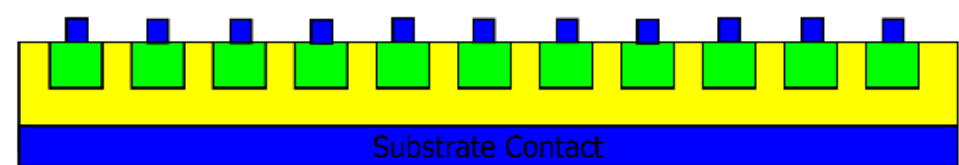

Figure 4. Structure of the realized photodetector: (a) top view; (b) cross section (figure not in scale).

In order to convert the current signal into a voltage signal, it is necessary to use a transimpedance amplifier (TIA), designed according to the schematic depicted in Figure 5.

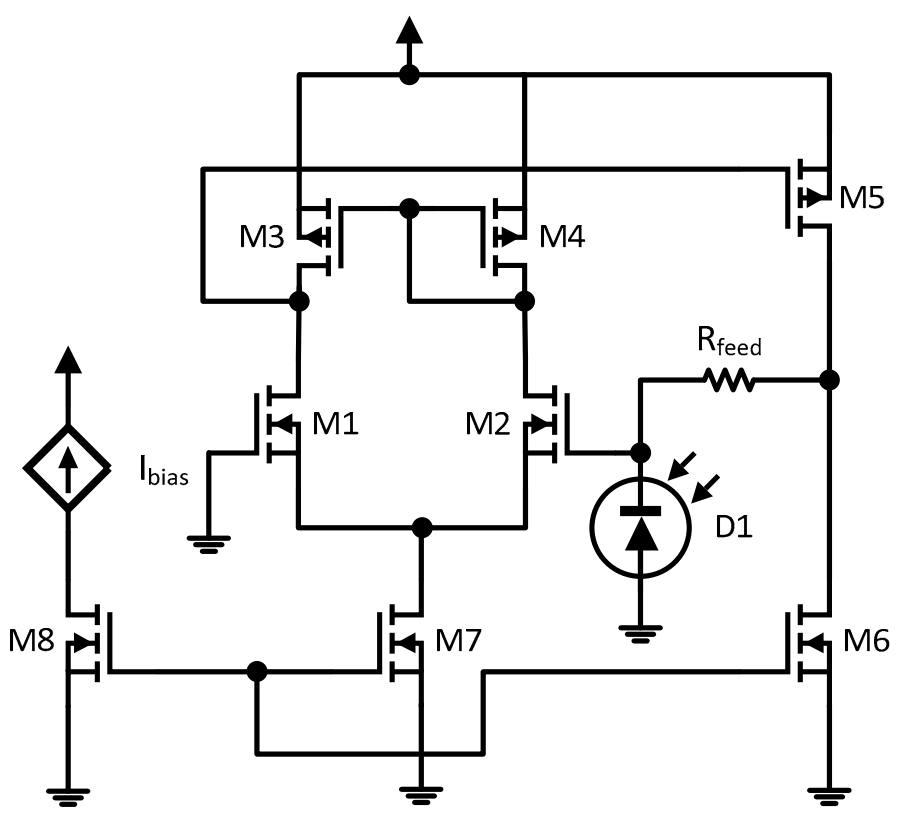

Figure 5. Integrated light sensor with transimpedance amplifier.

The integrated solar cell area $\left(300 \times 200 \mu \mathrm{m}^{2}\right)$ was chosen to generate a photocurrent in a range that suits the linear region of the amplifier (up to $22 \mu \mathrm{A}$ ). By assuming an indicative maximum efficiency of $10 \%$, the area was chosen in such a way that under $1000 \mathrm{~W} / \mathrm{m}^{2}$ the cell would still provide a photocurrent within the said limits. The TIA is based on an integrated MOSFET operational amplifier. This operational amplifier performs an open loop gain of $100 \mathrm{~dB}$ with an output resistance of $0.3 \Omega$ and can operate in the temperature range from $-40{ }^{\circ} \mathrm{C}$ to $120^{\circ} \mathrm{C}$. The simulated TIA output is shown in Figure 6. The feedback resistor $R_{\text {feed }}$ value is $100 \mathrm{k} \Omega$. 


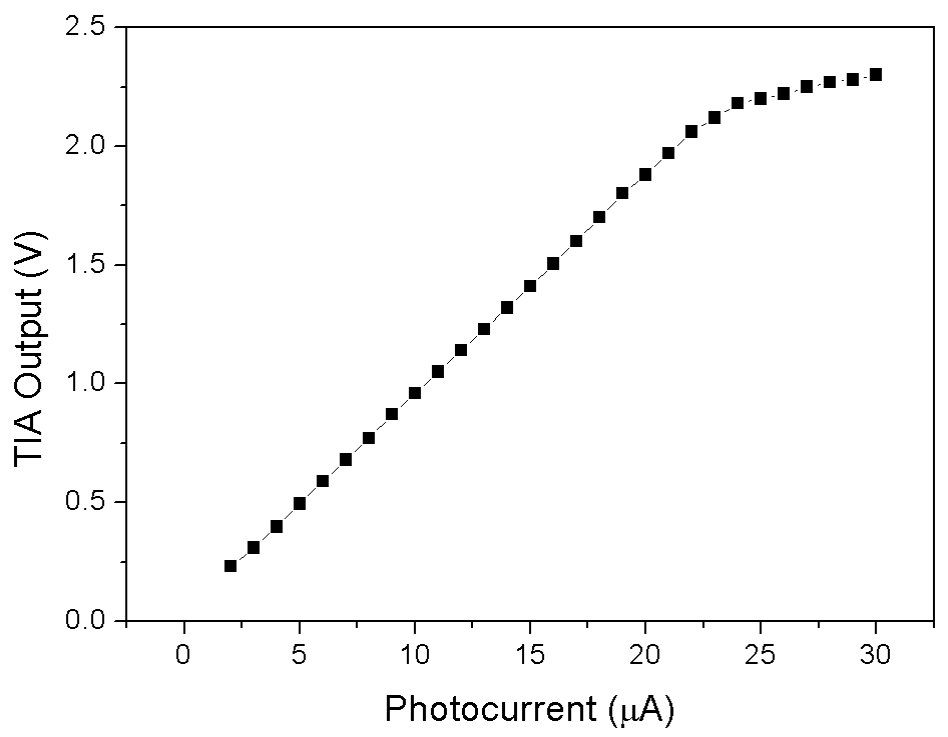

Figure 6. Simulated characteristic of the transimpedance amplifier output vs photocurrent.

\subsection{Clock}

Conventional current-starved single-ended ring oscillators are usually employed for realizing wide tuning range, voltage controlled oscillators that act as local clock for the digital circuitry of ASICs by exploiting the dependence of the large signal oscillation frequency $f_{\text {Osc }}$ on the bias current $I_{b}[15,16]$ that is determined by:

$$
f_{\mathrm{Osc}}=\frac{1}{2 N t_{D}} \approx \frac{I_{b}}{2 N O s c C^{\prime}}
$$

where $N$ is the number of inverters used, $t_{D}$ the time delay of each stage, $V_{\text {Osc }}$ the oscillation amplitude and $C$ the load capacitance.

Furthermore, in a ring oscillator topology the small-signal amplitude of the oscillating signal can be expressed as

$$
V_{\text {Out }}(t)=v_{\text {Out }}(0) \exp \left(\frac{\left|A_{s}\right|-2}{2} \omega_{s} t\right) \cos \left(\frac{\left|A_{s}\right| \sqrt{3}}{2} \omega_{s} t\right)
$$

where $v_{\mathrm{Out}}(0)$ is the initial condition at the ring oscillator output, $A_{s}$ the gain of each amplifier stage and $\omega_{s}$ the $-3 \mathrm{~dB}$ bandwidth of each stage [13]. The first term of (5) represents the time-varying amplitude of the oscillations. Therefore, the settling time can be expressed to a first approximation as

$$
t_{s}=\frac{2}{\left(\left|A_{s}\right|-2\right) \omega_{s}} \ln \left(\frac{0.9 V_{\text {Osc }}}{v_{\text {Out }}(0)}\right)
$$

The settling time of a ring oscillator ( $\mathrm{RO})$, at a given initial condition, depends only on the amplifier stages characteristics (gain and bandwidth). In particular, when the oscillator is implemented by exploiting CMOS inverters, the small-signal gain of a single stage is given by

$$
A_{s}=-\left(\mathrm{g}_{m_{n}}+\mathrm{g}_{m_{p}}\right) r_{o_{n}} \| r_{o_{p}}
$$

where $r_{o n}$ and $r_{o p}$ are the output resistances of the MOS devices. However, an increase of the transconductance of the inverting stages does not necessarily imply a reduction of the oscillator settling time because a higher gain requires more current consumption and thus larger size components with increased parasitics that tend to decrease $\omega_{s}$. To obtain a fast start-up oscillator, required for low power clocks, bias current and hence devices sizes have to be properly set. 
In this work, the oscillator provides a stable carrier of $10 \mathrm{MHz}$ frequency $\left(W_{p} / W_{n}=4, C_{1}=C_{2}=\right.$ $C_{3}=790 \mathrm{pF}$ ). For achieving this purpose, the same bandgap current reference introduced in Section 3.1 has been used, providing high robustness against supply voltage and temperature fluctuations. A clock signal of $5 \mathrm{kHz}$ is thus obtained using a frequency divider $\left(f_{\text {in }} / f_{\text {out }}=2000\right)$.

\subsection{Digital Section}

In Figure 7 the blocks of the digital section are shown. An ADC was used to convert the analogue signals produced by the sensors; a parallel-in-serial-output (PISO) shift register was implemented to serialize the parallel output of the ADC; a Load signal is necessary to activate the loading by the PISO. In this work, an integrated 8-bit successive approximation ADC was used, available in the standard cell library of the AMS technology.

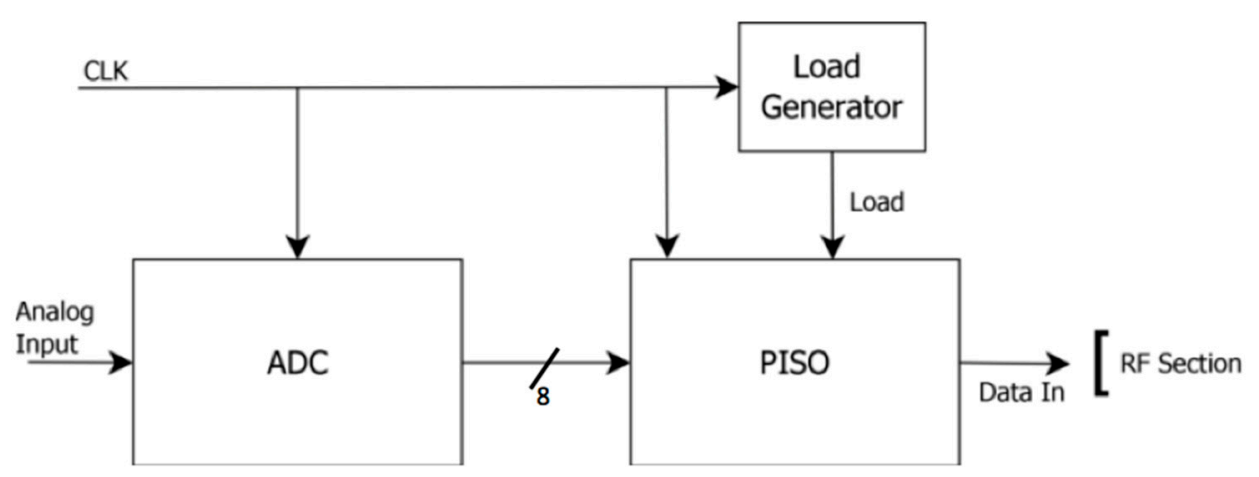

Figure 7. Block diagram of the Digital Section.

The PISO output normally consists of $4 \times 8$-bit words. Five words are used however when the external sensor is also selected. The first and the last words are used to identify Start and Stop of modulation. They can additionally contain a microchip read-only identifier. The entire sequence of bits is depicted in Figure 8. Between the first and the last 8-bits, there are the digital values of the PTAT and light sensor (payload).

\section{$01010101 \mid$ PTAT Data $\mid$ Light Sensor Data $\mid 10101010$}

Figure 8. Serialized output of 32-bits PISO.

The Load command generator was designed to generate a high-level every 50 cycles of the $5 \mathrm{kHz}$ Clock.

\subsection{RF Section}

In this microchip sensor, a directly On-Off-Keying (OOK) modulated oscillator-based transmitter was used, as depicted in Figure 9. The transmitter topology exploits a cross-coupled complementary LC oscillator running at a frequency of $2.5 \mathrm{GHz}$. The differential output is coupled to a common source differential power amplifier and buffered on an integrated dipole antenna. A start-up circuit allows the duty cycling of the transmitter and the implementation of the OOK modulation. 


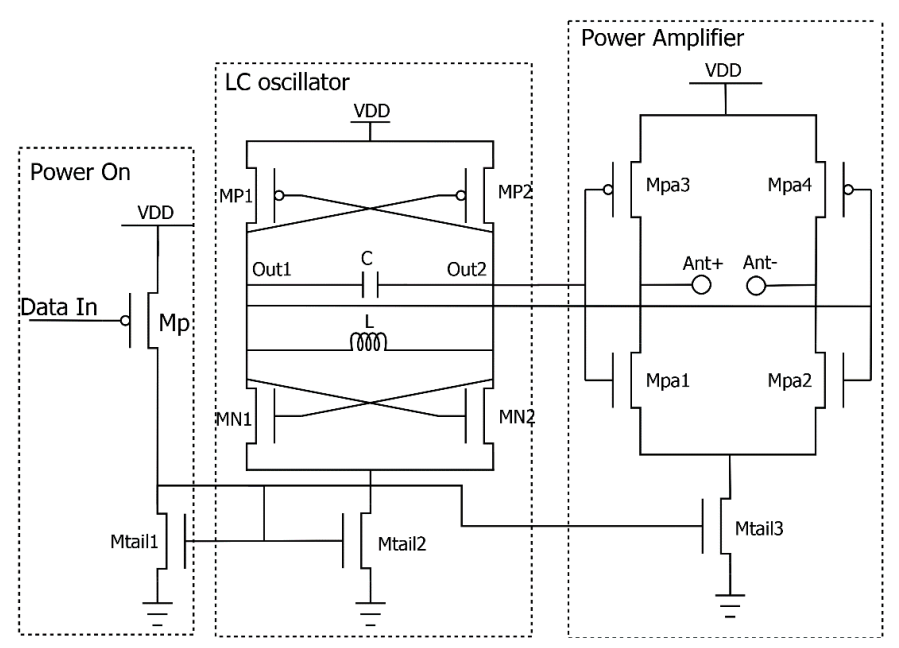

Figure 9. Circuit schematic of the implemented transmitter.

LC cross-coupled oscillators are suitable circuits to be used in radio frequency systems. These kinds of oscillators offer high frequency stability over temperature, voltage and process tolerances and low phase noise [13].

In this oscillator topology, the oscillation frequency is set by an inductors-capacitors (LC) network according to the formula:

$$
f=\frac{1}{2 \pi \sqrt{\mathrm{LC}}}
$$

Despite the simplicity of the cross-coupled differential scheme, the complementary architecture features a higher negative transconductance useful for overcoming the resonating circuit losses, essentially due to the parasitic resistance of the LC tank and sustaining oscillations [17]. In particular the following relationship must be verified [18]:

$$
\frac{2}{G_{m}} \geq R_{t a n k}
$$

where $G_{m}$ is the transconductance provided by the differential couple and $R_{\text {tank }}$ is the parasitic resistance of the LC tank.

The complementary topology allows oscillations to start also for low quality factors $\left(Q_{\operatorname{tank}}\right)$ of the resonating circuits, which is typical for fully integrated oscillators. In fact, the inductor shows a poor quality factor $Q_{L}$, typically in a range $4-20$ for enhanced structures $[19,20]$.

The resonating circuit employs $\mathrm{L}=4 \mathrm{nH}$ and $\mathrm{C}=1 \mathrm{pF}$. In particular, we used 4-turns square spiral inductors fabricated in the top aluminium metal layer and polysilicon capacitors. The employed coils exhibit a quality factor of 4.7 at $2.5 \mathrm{GHz}$ [9].

Each transistor has a $\mathrm{W} / \mathrm{L}$ ratio set to achieve the needed negative transconductance for sustaining oscillations. Furthermore, the sizes of the cross-coupled pairs, together with the current tail, were chosen to properly bias the differential power amplifier. Table 1 summarizes the key dimensions of each transistor.

Table 1. Transistor dimensions of differential cross coupled LC oscillator.

\begin{tabular}{cccccc}
\hline & MP1 & MP2 & MN1 & MN2 & Mtail \\
\hline $\mathbf{W}$ & $72 \mu \mathrm{m}$ & $72 \mu \mathrm{m}$ & $72 \mu \mathrm{m}$ & $72 \mu \mathrm{m}$ & $100 \mu \mathrm{m}$ \\
$\mathbf{L}$ & $0.35 \mu \mathrm{m}$ & $0.35 \mu \mathrm{m}$ & $0.35 \mu \mathrm{m}$ & $0.35 \mu \mathrm{m}$ & $0.35 \mu \mathrm{m}$ \\
\hline
\end{tabular}


A linear power amplifier (PA) based on a class B bridge configuration amplification stage was implemented to feed the small dipole antenna. The differential MOSFET pairs were accurately sized for maximizing the power delivered to the antenna alternatively through the Mpa3-Mpa2 and Mpa4-Mpa1 paths. A W/L ratio of $65 \mu \mathrm{m} / 0.35 \mu \mathrm{m}$ was set for all Mpa transistors. The estimated power gain is $2.3 \mathrm{~dB}$ with a total harmonic distortion (THD) of $0.13 \%$, estimated from simulations. This stage is turned on by Mtail3 only during transmission.

The on-chip dipole radiating element is made within the top aluminium metal layer, having a thickness of $0.925 \mu \mathrm{m}$. This antenna requires a differential excitation, which suits the PA scheme.

For a resonating antenna, the linear dimensions must be of the order of one half of the carrier wavelength. Therefore, for operations at $2.4 \mathrm{GHz}$, a well-tuned dipole antenna should have dimensions of the order of centimetres. However, the chip size limits the antenna dimension, so it is not possible even approaching the resonating conditions in our case. The characteristic parameters of such a small antenna are obviously very poor, with an estimated gain of about $-50 \mathrm{~dB}$ and a radiation efficiency of the order of $1 \%$ [21], enough however to allow short range transmissions [6].

\section{Experimental Results}

The circuital sections were first separately characterized. In particular, tests were made on the analogue sensors (temperature and light), the digital conversion section with data serialization and finally on the RF transmitter.

A microphotograph of the die is shown in Figure 10a. The area of the realized chip is $1 \mathrm{~mm} \times 4 \mathrm{~mm}$. After fabrication, for a complete characterization, the test chips were packaged in 44-pin TQFP packages (Figure 10b) which give easy access to all input and output nodes of the circuit. Bare dies samples were also tested after ultrasonic wire bonding to a limited number of bondpads, e.g., for RF transmission tests.

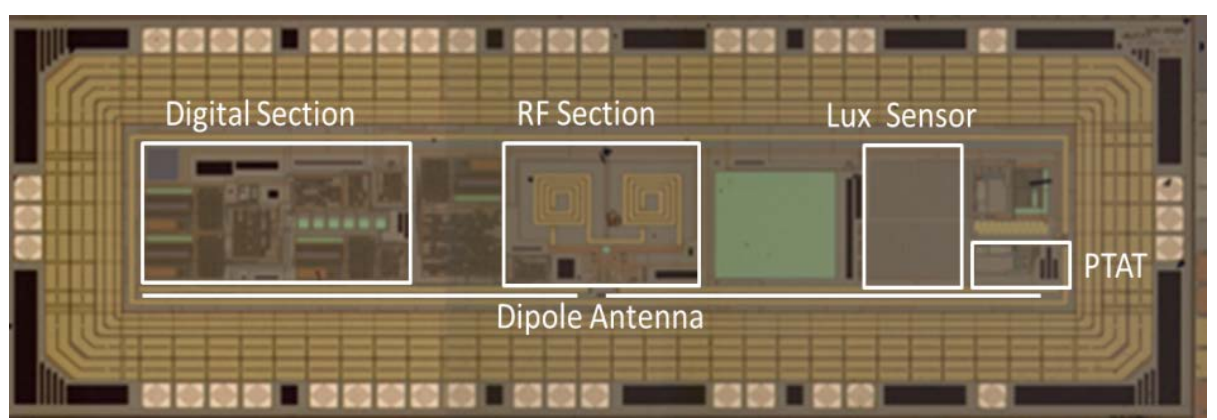

(a)

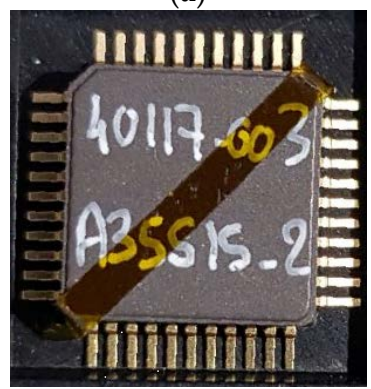

(b)

Figure 10. (a) Microphotograph of the realized chip. The main sections of the microchip are highlighted; (b) Microphotograph of an encapsulated microchip.

\subsection{Temperature Sensor}

To investigate the performance of the PTAT sensor, the whole chip was tested in a thermostatic oven, using a standard PT100 as reference as shown in Figures 11 and 12. 


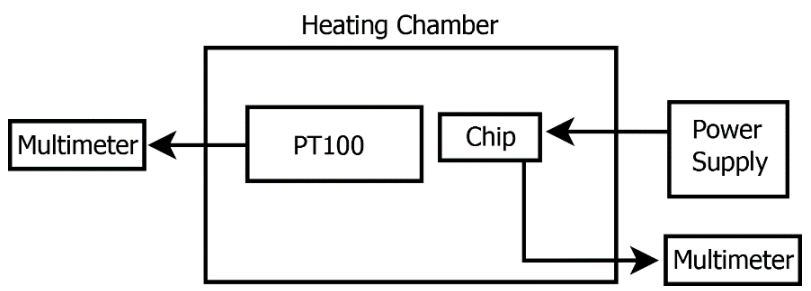

Figure 11. Experimental setup used to characterize the PTAT performances.

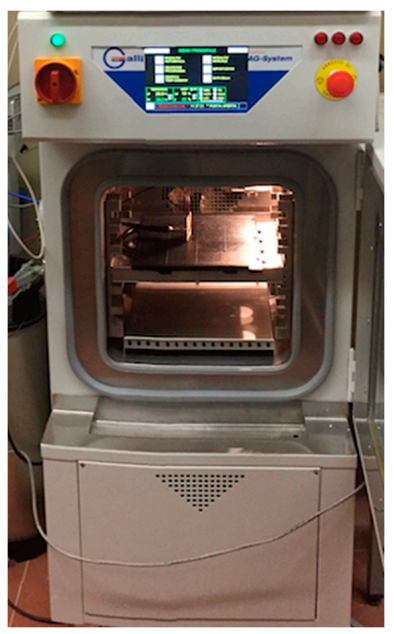

Figure 12. Picture of the setup used for the characterization of the temperature sensor.

As shown in Figure 13, the integrated PTAT presents a good linearity $\left(R^{2}=0.99962\right)$ in the temperature range of interest $\left(20{ }^{\circ} \mathrm{C}-90{ }^{\circ} \mathrm{C}\right)$ with a sensitivity of $19.1 \mathrm{mV} /{ }^{\circ} \mathrm{C}$, measured at the analogue amplifier output. Inside the climatic chamber it was possible to change in a small range the lighting conditions in combination with temperature. In particular, a modification of the LED array output, from 10 to $50 \mathrm{~W} / \mathrm{m}^{2}$ approximately, was applied showing no effect at all on the temperature sensor output. However, it can be expected that higher irradiations would induce heating of the device.

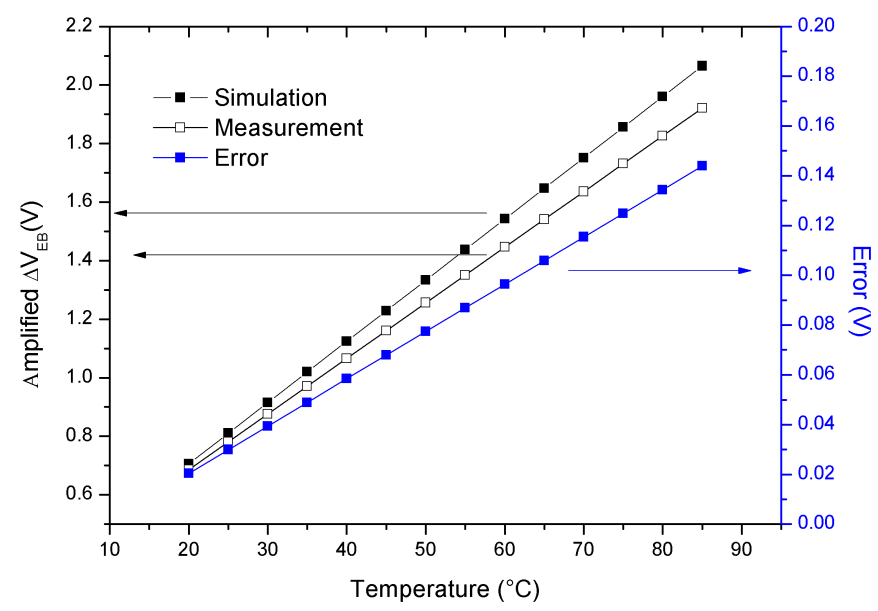

Figure 13. Experimental characteristic of the PTAT sensor, obtained using analogue output. The simulated characteristic is also provided for comparison. The error between simulated and experimental characteristic is also added.

This specific implementation of the sensor does not include a calibration circuitry, which could be however obtained in a final version by e.g., laser trimming acting on $\mathrm{I}_{\text {ref }}$ or other standard techniques.

Table 2 summarizes the PTAT key parameters. 
Table 2. Performance summary of proposed PTAT sensor.

\begin{tabular}{cccccc}
\hline Power Supply (V) & $\begin{array}{c}\text { Drawn } \\
\text { Current }(\boldsymbol{\mu A})\end{array}$ & $\begin{array}{c}\text { Area } \\
\left(\mathrm{mm}^{2}\right)\end{array}$ & $\begin{array}{c}\text { Temperature } \\
\text { Range }\left({ }^{\circ} \mathbf{C}\right)\end{array}$ & $\begin{array}{c}\text { Sensitivity } \\
\left(\mathbf{m V} /{ }^{\circ} \mathbf{C}\right)\end{array}$ & Linearity \\
\hline 3.3 & 150 & 0.022 & $25-90$ & 19.1 & 0.99962 \\
\hline
\end{tabular}

\subsection{Light Sensor}

To examine the performance of the light sensor, the whole chip was exposed to direct sunlight and the photocurrent was measured at several irradiations (IRR), continuously monitored with a hand-held solar power meter. Photocurrent has been measured using an Agilent Technologies 4155C Semiconductor parameter analyser.

As shown in Figure 14 the light sensor presents a good linearity $\left(R^{2}=0.99915\right)$ in the range of interest of a typical sunny day. The sensitivity is calculated as follows:

$$
S_{\text {Cel+Tia }}=\frac{\Delta V_{T I A}}{\Delta I R R}=16.96 \mathrm{mV} / \mathrm{w} / \mathrm{m}^{2},
$$

In this specific implementation of the sensor, an additional calibration feature was not considered, though it would be possible by e.g., laser trimming of $R_{\text {feed }}$.

Temperature variations can affect the light sensor output. This dependence is shown in Figure 15, which demonstrates that, due to the amplifier non-idealities, the light reading saturates at lower and lower photocurrent values as the temperature increases.

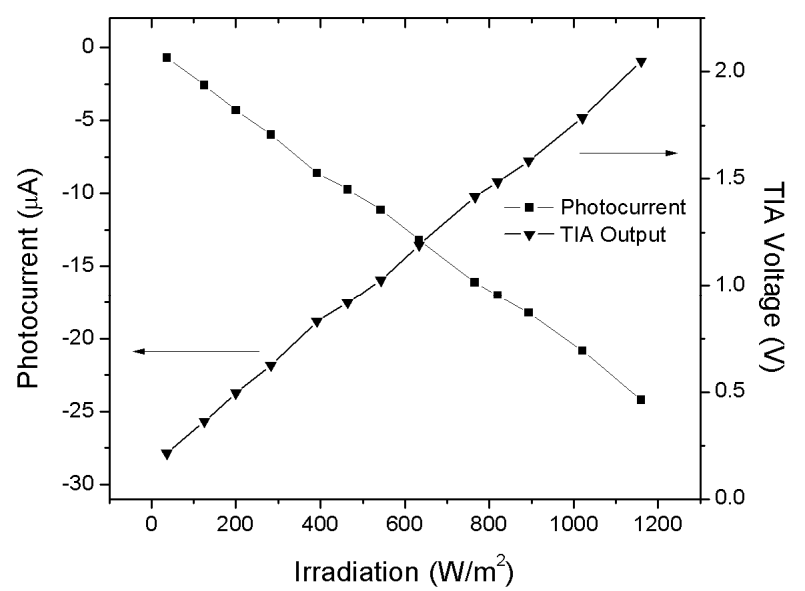

Figure 14. Characteristic of the irradiation sensor, obtained using analogue output.

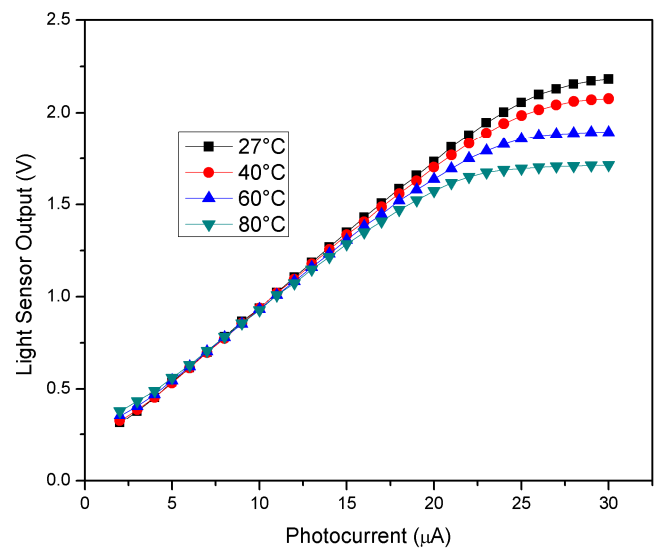

Figure 15. Light sensor output vs. photocurrent at different temperatures. 


\subsection{RF Transmitter Performance}

The RF performance of the implemented transmitter was characterized by analysing the transmitted signal received by a $0.4-3 \mathrm{GHz}$ bandwidth, $5 \mathrm{dBi}$ logarithmic periodic dipole antenna (model Rhode\&Schwarz HL040) connected in turn to a Rhode\&Schwarz FSV Spectrum Analyser. The antenna was placed at a distance of $1 \mathrm{~m}$ from the microchip.

The experimental measurements show a continuous wave $(\mathrm{CW})$ signal, produced by the cross-coupled LC oscillator, at about $2.54 \mathrm{GHz}$ as shown in Figure 16.

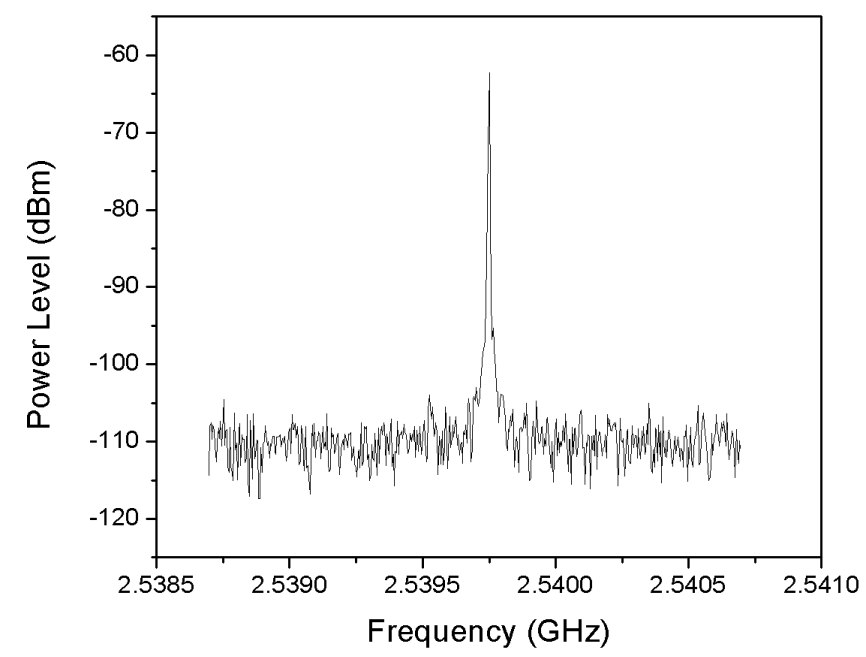

Figure 16. Frequency spectrum graph of the received signal.

The proposed LC oscillator shows a phase noise of $-90 \mathrm{dBc} / \mathrm{Hz}$ at $1 \mathrm{MHz}$ offset from the centre frequency, as shown in Figure 17. The phase noise is estimated as follows:

$$
L(\Delta f)_{d B c}=P_{S}(d B m)-P_{c}(d B m)-10 \log _{10}\left(\frac{R B W}{1 \mathrm{~Hz}}\right)
$$

where $L(\Delta f)_{d B c}$ is the phase noise, $P_{s}$ is the noise power level in a band of $1 \mathrm{~Hz}$ at a certain offset frequency from the carrier central frequency $f_{0}, P_{c}$ is the power level of the carrier at $f_{0}$ and RBW is the bandwidth resolution of the spectrum analyser.

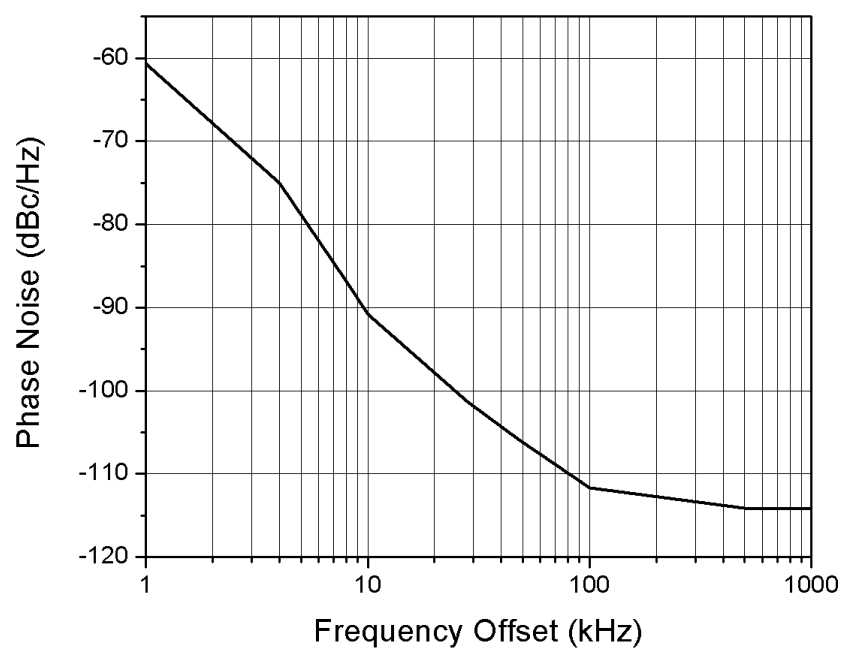

Figure 17. Phase noise of the received signal.

The LC transmitter shows a temperature sensitivity $\Delta f / \Delta T=-0.25 \mathrm{MHz} /{ }^{\circ} \mathrm{C}$. 


\subsection{Whole System Operation}

The operation of the whole system was tested employing a commercial hand-held IC-R20 (Icom America Inc., 12,421 Willows Road NE, Kirkland, WA, USA) receiving unit placed at a distance of $1 \mathrm{~m}$, although the transmitted RF signal was clearly detectable up to a distance of $1.5 \mathrm{~m}$ (Table 3). The assembled device was tested under the sun at a temperature of $24{ }^{\circ} \mathrm{C}$ and an irradiation level of $1125 \mathrm{~W} / \mathrm{m}^{2}$. In agreement with the previous characterization, the output voltage of the PTAT and light sensor were $0.763 \mathrm{~V}$ and $1.971 \mathrm{~V}$ respectively.

Table 3. Comparison table with state-of-the-art sensors.

\begin{tabular}{cccccc}
\hline Ref. & $\begin{array}{c}\text { Area } \\
(\mathbf{m m} \times \mathbf{m m})\end{array}$ & $\begin{array}{c}\text { Antenna } \\
\text { Type }\end{array}$ & $\begin{array}{c}\text { Reading } \\
\text { Distance }\end{array}$ & $\begin{array}{c}\text { Temperature } \\
\text { Range }\left({ }^{\circ} \mathbf{C}\right)\end{array}$ & $\begin{array}{c}\text { Additional } \\
\text { Sensors }\end{array}$ \\
\hline This work & $1 \times 4$ & Dipole & $1.5 \mathrm{~m}$ & $25-90$ & light \\
U. Calpa et al. [5] & $1.5 \times 1.5$ & Inductor & $110 \mathrm{~cm}$ & $15-35$ & no \\
Boram Kim et al. [22] & $7.5 \times 7.5$ & Inductor & $5 \mathrm{~mm}$ & $24-40$ & no \\
Arsalan et al. [8] & $3.2 \times 1.5$ & Spiral & $3 \mathrm{~cm}$ & $27-35$ & no \\
Jun Yin et al. [23] & $0.9 \times 1.25$ & External & $4 \mathrm{~m}$ & $-20-30$ & no \\
\hline
\end{tabular}

The transmitted data bitstream is shown in Figure 18 (top) together with the recorded signal at the output of the radio receiver (bottom). Figure 18 (bottom) shows signal stability issues that could be suppressed by properly designing the AC-coupling of the demodulated signal to a low-frequency (audio) amplifier instead of that integrated in the used commercial receiver. The received values are $23.4^{\circ} \mathrm{C}, 1118 \mathrm{~W} / \mathrm{m}^{2}$.
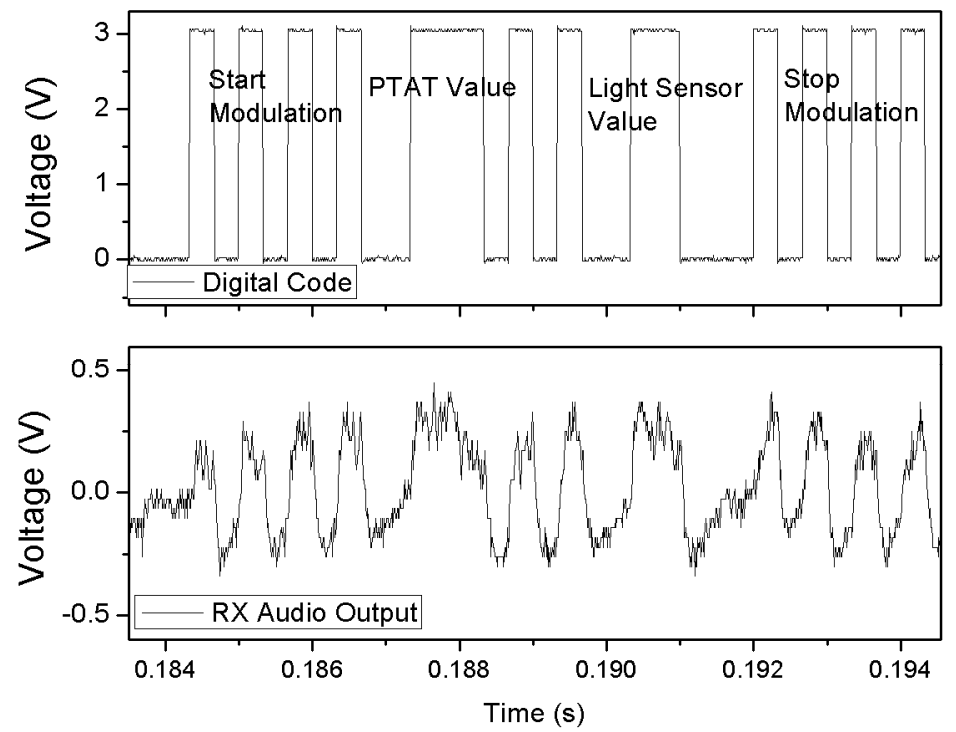

Figure 18. Sample transmitted code (top) and demodulated signal (bottom) as recorded at the output of the receiver. The bit stream contains the data produced by the PTAT and the light sensors.

\section{Conclusions}

In this paper, a full custom microchip realized in $0.35 \mu \mathrm{m}$ CMOS technology, designed for ambient and processes monitoring, was proposed. It features a temperature and an illumination sensor. The temperature sensor features a sensitivity of $19.1 \mathrm{mV} /{ }^{\circ} \mathrm{C}$ and a linearity 0.99962 in the temperature range of $20{ }^{\circ} \mathrm{C}$ to $90{ }^{\circ} \mathrm{C}$, while the illumination sensor, designed to operate in the range from 0 to $1200 \mathrm{~W} / \mathrm{m}^{2}$, shows a sensitivity of $16.96 \mathrm{mV} / \mathrm{W} / \mathrm{m}^{2}$ with a linearity of 0.99915 .

An integrated $2.5 \mathrm{GHz}$ transmitter with an On-Chip-Antenna (OCA)-made in the top metal layer of the same die-transmits the collected data wirelessly by means of an on-off amplitude modulation 
scheme, at a bit rate of $3 \mathrm{kbit} / \mathrm{s}$. The low bit rate was chosen in order to obtain that the decoded signal at the receiver output were in the audible band, as the receiver is a commercial low-cost radio receiving unit. However, simulations indicate that bit rates as high as $100 \mathrm{kbit} / \mathrm{s}$ could be easily obtained with the same technology.

Acknowledgments: This work was partially funded by the Italian Government-Ministry of Economic Development, under the call "Progetti di Innovazione Industriale per l’Efficienza Energetica", Project code C31J09000420005.

Author Contributions: M.M. and C.F. conceived and designed the microsensor and performed the experimental tests; F.G.D.C. conceived the experiment and supervised the design. All authors contributed equally in writing the paper.

Conflicts of Interest: The authors declare no conflict of interest.

\section{References}

1. Atzori, L.; Iera, A.; Morabito, G. The Internet of Things: A survey. Comput. Netw. 2010, 54, 2787-2805. [CrossRef]

2. Eder, C.; Valente, V.; Donaldson, N.; Demosthenous, A. A CMOS smart temperature and humidity sensor with combined readout. Sensors 2014, 14, 17192-17211. [CrossRef] [PubMed]

3. Rekhi, A.S.; Khuri-Yakub, B.T.; Arbabian, A. Wireless Power Transfer to Millimeter-Sized Nodes Using Airborne Ultrasound. IEEE Trans. Ultrason. Ferroelectr. Freq. Control 2017, 64, 1526-1541. [CrossRef] [PubMed]

4. Zaid, J.; Abdulhadi, A.; Kesavan, A.; Belaizi, Y.; Denidni, T.A. Multiport circular polarized RFID-tag antenna for UHF sensor applications. Sensors 2017, 17, 1576. [CrossRef] [PubMed]

5. Unigarro Calpa, E.; Achury Florian, A.; Rairez Rodriguez, F.; Sacristan, J.; Bohorquez, J.; Segura-Quijano, F.E. Towards Fully-integrated Low-Cost Inductive Powered CMOS Wireless Temperature Sensor. IEEE Trans. Ind. Electron. 2017, 64, 8718-8727. [CrossRef]

6. Zito, F.; Aquilino, F.; Fragomeni, L.; Merenda, M.; Della Corte, F.G. CMOS wireless temperature sensor with integrated radiating element. Sens. Actuators A 2010, 158, 169-175. [CrossRef]

7. Aquilino, F.; Della Corte, F.G.; Merenda, M.; Zito, F. Fully-integrated wireless temperature sensor with on-chip antenna. In Proceedings of the 2008 IEEE Sensors, Lecce, Italy, 26-29 October 2008; pp. 760-763.

8. Arsalan, M.; Ouda, M.H.; Marnat, L.; Ahmad, T.J.; Shamim, A.; Salama, K.N. A 5.2 GHz, 0.5 mW RF powered wireless sensor with dual on-chip antennas for implantable intraocular pressure monitoring. In Proceedings of the IEEE MTT-S International Microwave Symposium Digest (MTT), Seattle, WA, USA, 2-7 June 2013. [CrossRef]

9. Datasheet AMS Foundry. 2017. Available online: http://asic.ams.com/ (accessed on 31 December 2017).

10. Bakker, A.; Huijsing, J.H. Micropower CMOS temperature sensor with digital output. IEEE J. Solid-State Circuits 1996, 31, 933-937. [CrossRef]

11. Meijer, G.C.M.; Wang, G.; Fruett, F. Temperature Sensors and Voltage References Implemented in CMOS Technology. IEEE Sens. J. 2001, 1, 225-234. [CrossRef]

12. Pertijs, M.A.P.; Meijer, G.C.M.; Huijsing, J.H. Precision Temperature Measurement Using CMOS Substrate PNP Transistors. IEEE Sens. J. 2004, 4, 294-300. [CrossRef]

13. Razavi, B. Design of Analog CMOS Integrated Circuits; McGraw-Hill: New York, NY, USA, 2001; Volume 1, p. 684.

14. Yoo, C.; Park, J. CMOS current reference with supply and temperature compensation. Electron. Lett. 2007, 43, 1422. [CrossRef]

15. Zhao, X.; Chebli, R.; Sawan, M. A wide tuning range voltage-controlled ring oscillator dedicated to ultrasound transmitter. In Proceedings of the 16th International Conference on Microelectronics, Tunis, Tunisia, 6-8 December 2004; pp. 313-316. [CrossRef]

16. Sun, L.; Kwasniewski, T.A. A 1.25-GHz 0.35- $\mu$ m monolithic CMOS PLL based on a multiphase ring oscillator. IEEE J. Solid-State Circuits 2001, 36, 910-916. [CrossRef]

17. Hajimiri, A.; Lee, T.H. Design issues in CMOS differential LC oscillators. IEEE J. Solid-State Circuits 1999, 34, 717-724. [CrossRef]

18. Bunch, R.; Raman, S. A 0.35 pm CMOS 2.5 GHz Complementary -GM VCO Using PMOS Inversion Mode Varactors. In Proceedings of the IEEE Radio Frequency Integrated Circuits (RFIC) Symposium, Phoenix, AZ, USA, 20-22 May 2001; Digest of Papers; pp. 49-52. 
19. Haobijam, G.; Paily, R. Quality factor enhancement of CMOS inductor with pyramidal winding of metal turns. In Proceedings of the 2007 International Workshop on Physics of Semiconductor Devices, Mumbai, India, 16-20 December 2007; pp. 729-732. [CrossRef]

20. Craninckx, J.; Steyaert, M.S. A $1.8 \mathrm{GHz}$ low phase-noise CMOS VCO using optimized hollow spiral inductors. IEEE J. Solid-State Circuits 1997, 32, 736-744. [CrossRef]

21. Aquilino, F.; Della Corte, F.G. On-Chip Integrated Antenna Structures for Biomedical Implantable Sensors. Procedia Chem. 2009, 1, 513-516. [CrossRef]

22. Kim, B.; Nakazato, K. Dual data pulse width modulator for wireless Simultaneous Measurement of Redox Potential and Temperature using a Single RFID Chip. In Proceedings of the 19th IEEE International Conference on Electronics, Circuits and Systems, Seville, Spain, 9-12 December 2012; pp. 368-371. [CrossRef]

23. Yin, J.; Yi, J.; Law, M.K.; Ling, Y.; Lee, M.C.; Ng, K.P.; Gao, B.; Luong, H.C.; Bermak, A.; Chan, M.; Ki, W.H.; Tsui, C.Y.; Yuen, M. A system-on-chip EPC Gen-2 passive UHF RFID tag with embedded temperature sensor. IEEE J. Solid-State Circuits 2010, 45, 2404-2420. [CrossRef]

(C) 2018 by the authors. Licensee MDPI, Basel, Switzerland. This article is an open access article distributed under the terms and conditions of the Creative Commons Attribution (CC BY) license (http://creativecommons.org/licenses/by/4.0/). 